\title{
TIME FUNCTIONS APPROPRIATE FOR SOME AFTERSHOCKS OF THE POINT MUGU, CALIFORNIA EARTHQUAKE OF FEBRUARY 21, 1973
}

\author{
By David King and Donald V. Helmberger
}

\begin{abstract}
Broad-band recordings of aftershocks of the Pt. Mugu earthquake at small epicentral distances provided an excellent opportunity to test source models for small earthquakes. Simple events recorded at nearly vertical incidence produced a single $\boldsymbol{P}$-wave pulse of a duration of about $0.07 \mathrm{sec}$ and a somewhat more complicated $S$ wave with a slightly longer duration. Such events are consistent with a point dislocation source for which $Q_{\beta}=100$ or for which there is directivity with the fault breaking downward. We attribute the more usual complexities of small earthquake records to multiple events, some of which we observed, layering effects combined with greater epicentral distances, and scattering.
\end{abstract}

\section{INTRODUCTION}

Aftershocks of the Point Mugu earthquake provided an opportunity to measure the local displacements at relatively small epicentral distances. Within a few hours of the main event, we positioned four broad-band three-component trailer units in the epicentral area. Of the dozen or so shocks recorded in the following 2 weeks, a few events were well recorded on all three components at each station. In this study, we will examine these seismograms in terms of recent source models.

Small earthquakes recorded locally are generally quite complicated. That is, the $P$ waves are rather emergent followed by larger and longer period $S$ waves. The San Fernando aftershocks are typical examples as determined from a recent report of observations of the aftershocks by Molnar et al. (1974). Following the corner-frequency approach as applied by Molnar et al. (1974), one assumes that the first few seconds of motion represents the primary $(P)$ radiations and the next few seconds represents the shear $(S)$ contribution. On the average, a plot of the spectra generated from sections of time series of this type yields a lower corner frequency for the $S$-wave pulse than for the $P$-wave pulse. The above authors suggest a relatively simple circular dislocation model to explain this relative shift in corner frequencies. However, since the corresponding fault dimension is very small (usually less than $1 \mathrm{~km}$ ) and the source is assumed to radiate essentially simultaneously, one would expect to see two sharp pulses with the $S$ being slightly longer period. Their model explains the spectra but fails to explain the sequential information and does not predict realistic looking synthetic seismograms. How does one explain such complicated looking seismograms? Possible explanations are: (1) horizontal layers are producing wave-guide effects, (2) multiple events, (3) random scattering produced by irregularities in the medium, or perhaps all three.

For the case of shallow events, it is easy to show synthetically that shear-dominated surface waves trapped in the soft material near the Earth's surface can be quite important. For instance, Love waves can be larger than the direct arrival at ratios of source depth to epicentral distances greater than four, Helmberger (1974). For the case of deep events, at ratios less than one, it becomes much more difficult to explain complicated records in terms of propagational effects and one must resort to multiple events or perhaps scattering at the higher frequencies. 


\section{Selected ObSERVATIONS ANd Discussion}

Since we are primarily interested in getting an accurate determination of time functions appropriate for small earthquakes, we chose to analyze in detail two simple events that were recorded at very small epicentral distances (see SYC in Figure 1). Some observations of these events are displayed in Figures 2 and 3. The locations and fault-plane solutions are taken from Cambell et al. (1973). These two events are quite deep as indicated by their $(S-P)$ times. However, their epicenters are not well known because of lack of control to the west, but they are probably good to within several kilometers. The relatively small $P$

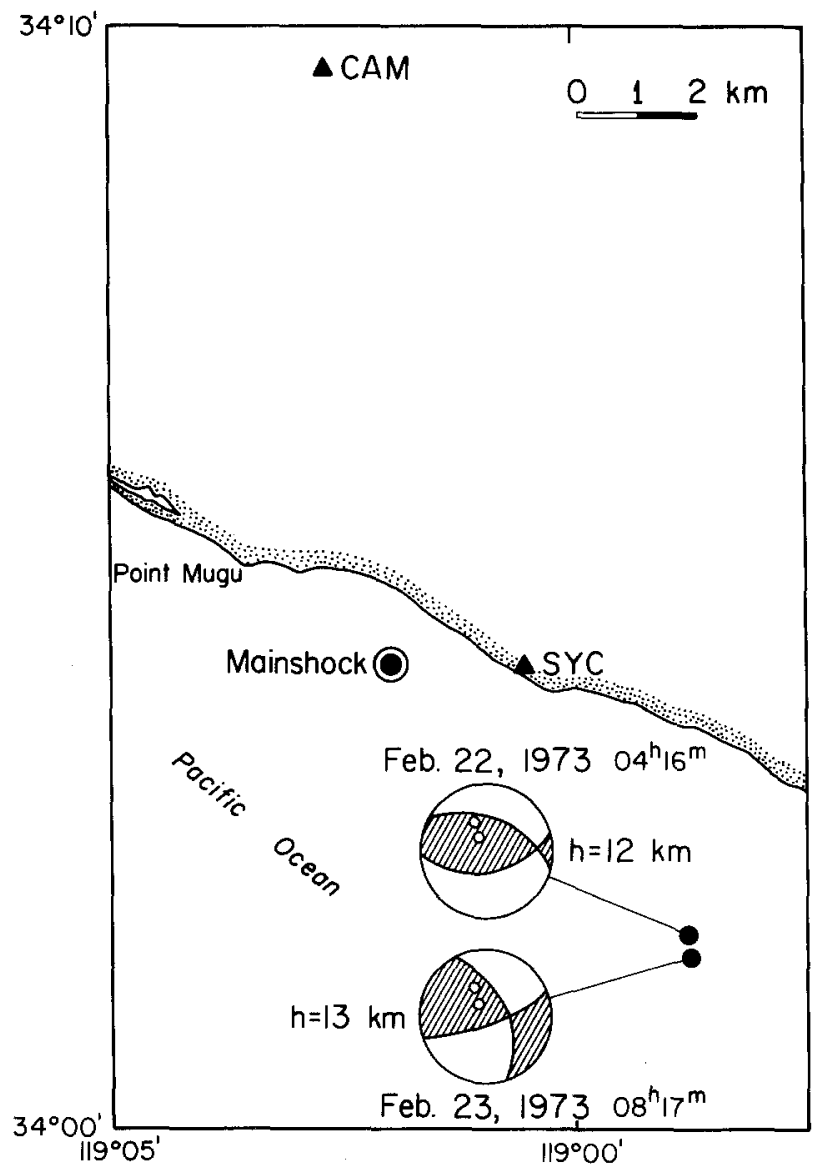

FIG. 1. Map showing the two events analyzed in detail and the stations used in this study. The fault plane solutions taken from Cambell et al. (1973) are for the upper half of the focal sphere. The open circles are the stations superimposed on the focal spheres.

component as measured on the horizontals at SYC indicates nearly vertical travel paths. Most of the energy of the $P$ and $S$ waves occur within their respective initial onsets at the SYC site; thus we will simply deconvolve the instrument response from these pulses to obtain the far-field time histories.

The recording system providing the data for this investigation uses a 5 -sec seismometer with a velocity transducer and writes on a FM analog tape. To provide dynamic range we record each displacement component on a low- and high-gain channel. The system response, excluding the playback effects, is given in Figure 4a. The dots were produced from shake-table tests. The playback recorder used to convert the FM signal to conventional seismograms introduces damping above $10 \mathrm{~Hz}$. This effect was determined by feeding in a 
step of voltage and obtaining a slightly smoothed step out. The total system response for a delta function in displacement is displayed in Figure $4 \mathrm{~b}$.

It appears from Figures 2 and 3 that the best estimate of $P$ radiation is obtained from the vertical component. On the other hand, it appears desirable to measure the $S$ radia-

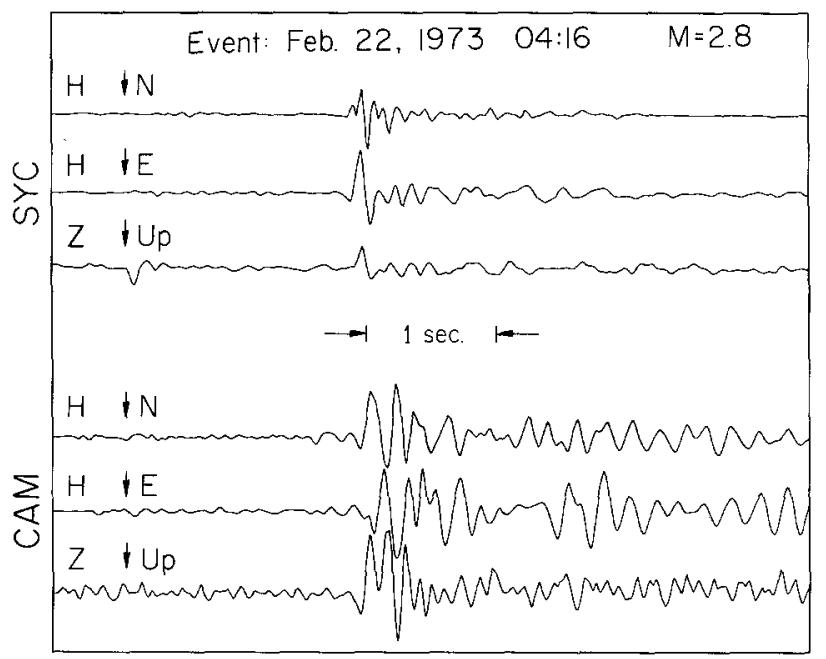

FIG. 2. The February 22 event as recorded at SYC and CAM. The gain of the CAM vertical componen was boosted by a factor of 3 during the playback to attempt to distinguish the $P$ arrival from the background noise. The north component as recorded at SYC does not contain the proper shaping filter and is essentially a high-frequency recording. The east and vertical traces are from a matched broad-band system.

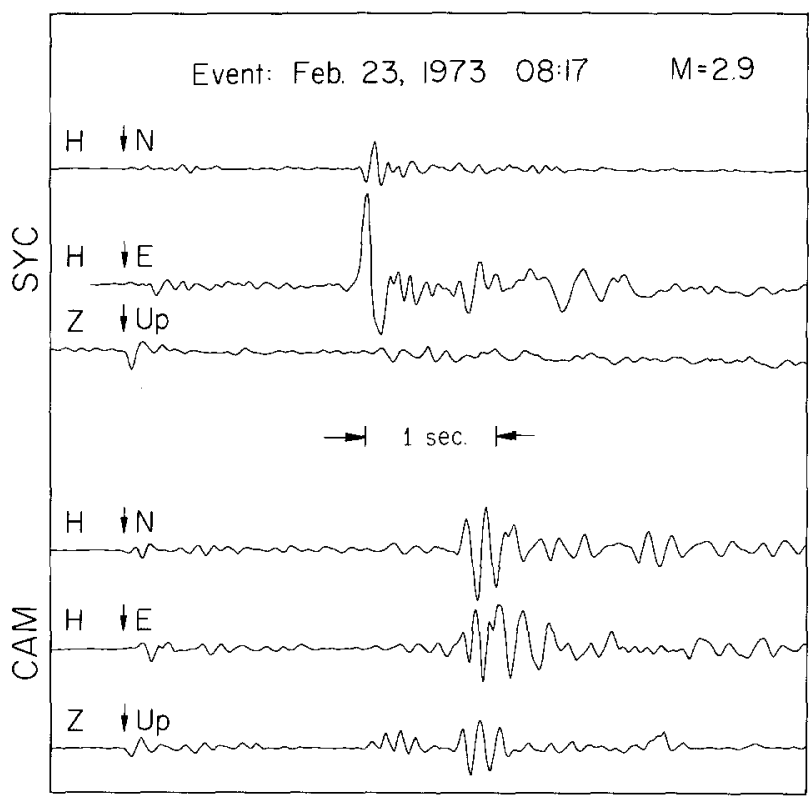

FIG. 3. The February 23 event as recorded at SYC and CAM.

tion from the horizontal component. The deconvolution operations for these two events with the corresponding observed wave forms are displayed in Figure 5. The deconvolved $P$ displacements are relatively simple with durations of about 0.06 to $0.08 \mathrm{sec}$. This should also be the duration of the major fault motions, ignoring the doppler shift caused by fault 


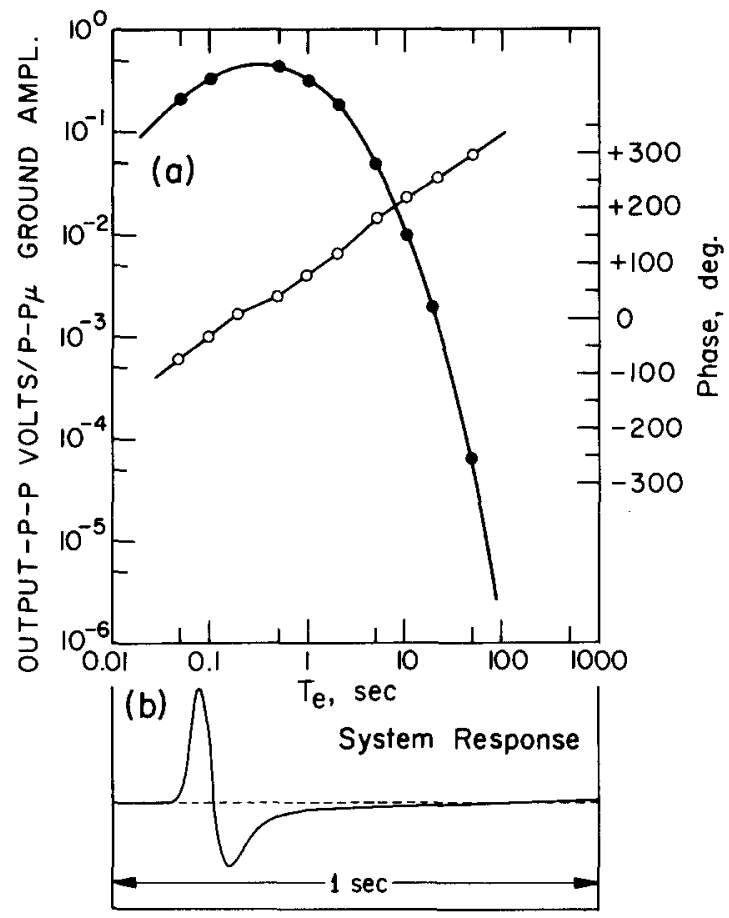

Fig. 4. (a) Frequency response of the seismometer system, not including the playback system. (b) Total system response including the playback system.

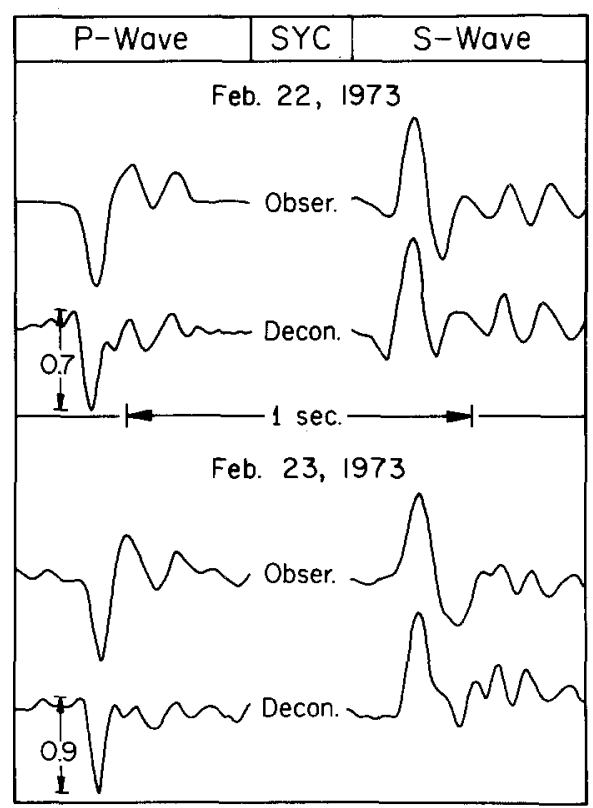

Fig. 5. Observed and deconvolved $P$ and $S$ wave forms for the two events. The calculated ground displacement for the $P$ arrivals is indicated in microns. 
breakage toward or away from the recording station. Assuming that the rupture velocity is no greater than the shear velocity limits the fault size to $0.5 \mathrm{~km}$ or less. The deconvolved $S$ displacements have slightly longer periods and are somewhat more complicated. If these events can be treated as point-source shear dislocations we would expect the $P$ and $S$ time histories to be identical, neglecting structure and absorption. Applying a $Q$-operator with $Q_{\alpha}=500$ and $Q_{\beta}=100$ produces roughly the same basic durations. However, we think $Q_{\beta}=100$ is somewhat low. Another interpretation is that the fault has some directivity (finiteness) with the dislocation propagating downward. Of course, it should be apparent that one cannot separate the dislocation history from finiteness given just one station. Unfortunately, the other observations are too complicated to sort out the true time histories from propagational effects. The observations at CAM, Figures 2 and 3 , appear to be the simplest of the more distant recordings. Here the distance is still too small to predict significant diving arrivals and the source is too deep to effectively couple energy into horizontal surface layering. However, a careful examination of the shear arrivals indicates a considerable train of slightly longer-period signals recorded on the horizontals

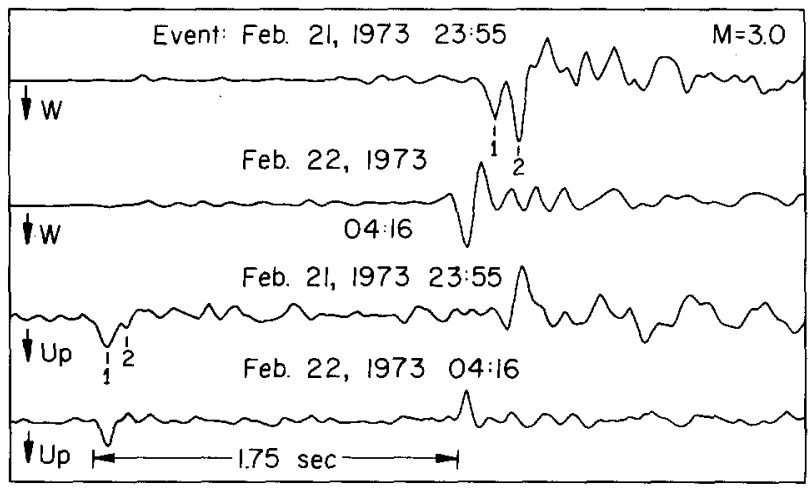

Fig. 6. Comparison of the double event of February 21 and the February 22 event as recorded at SYC. The two upper traces are the $S$ arrivals recorded on the E-W horizontal. The lower traces are the vertical components of the $P$ arrivals.

that does not appear on the verticals. One notices also that the $S$ arrival generally starts earlier on the verticals. These two features suggest that the incoming shear arrival interacts severely with the surface layerings. Perhaps an irregular layer could explain such effects. This would be consistent with the local geology at CAM which is on alluvium next to a volcanic outcrop.

The two events displayed in Figures 2 and 3 were the simplest recorded; other events at SYC appear somewhat more complicated, as seen by the example given in Figure 6 . The February 21 event appears to be nearly below the station and probably somewhat deeper than the February 22 event. However, by comparing the $P$ waves and corresponding $S$ waves, one gets the impression that this event is complex. Perhaps, we are dealing with stick-slip ruptures of the type observed by Brune (1973) in his foam rubber experiments. A similar conclusion on the complexity of deep earthquakes, depths greater than $500 \mathrm{~km}$, was reached by Burdick and Helmberger (1974).

To check the self-consistency of our records, we computed the expected first motions for $P$ and $S$ from the fault-plane solutions given by Cambell et al. (1973) for the events. We found that all components had the expected polarities for $P$, but not for $S$ where the north-south component of SYC was reversed from the expected polarity for the February $22,4: 16$ event. However, this station did have a weak $P$ arrival consistent with its nodal 
position on the fault plane. Because of uncertainties in location and in the fault-plane solution, we feel that our recordings are consistent within the limits of the errors involved.

\title{
CONCLUSIONS
}

Preliminary investigations of synthetics for models containing a surface layer indicate that significant wave-form distortions can occur at relatively small epicentral distances. These distortions can be avoided by obtaining observations at small ratios of epicentral distance to source depth, measuring the $P$ time history on the vertical and $S$ on the horizontals. Estimates of duration of about $0.07 \mathrm{sec}$ derived by deconvolution were obtained for two simple events with local magnitudes near three. There is some evidence for a slight elongation of the $S$ far-field time history relative to $P$. This effect can be explained by a $Q_{\beta}=100$ or directivity. Our results on the shift of $P$ corner frequency (signal duration) relative to $S$ are not as dramatic as those of Molnar et al. (1974). Furthermore, our observations of double events coupled with the lengthening of the shear wave train for even small increases in epicentral distance suggest that corner frequencies derived from major portions of the $P$ and $S$ wave trains may be misleading.

\section{ACKNOWLEDGMENTS}

The authors were assisted by Francis Lehner, Len Blayney, John Lower, and Dan Tanner in the data collection and in the detailed synthesis of the system response.

This research was supported by the Advanced Research Projects Agency of the Department of Defense and was monitored by the Air Force Office of Scientific Research under Contract FF44620-72-C-0083.

\section{REFERENCES}

Burdick, L. J. and D. V. Helmberger (1974). Time functions appropriate for deep earthquakes, Bull. Seism. Soc. Am. 64, 1419-1427.

Brune, J. N. (1973). Earthquake modeling by stick-slip along precut surfaces in stressed foam rubber, Bull. Seism. Soc. Am. 63, 2105-2119.

Cambell, R. H., W. L. Ellsworth, D. P. Hill, R. A. Page, M. D. Woods, R. W. Alewine, T. C. Hanks, T. H. Heaton, J. A. Hileman, H. Kanamori, B. Minster, and J. H. Whitcomb (1973). Point Mugu, California earthquake, 21 February 1973, and its aftershocks, Science, 182, 1127-1129.

Helmberger, D. V. (1974). Modeling local earthquakes as shear dislocations in a layered half-space, (in preparation).

Molnar, P., B. E. Tucker, and J. N. Brune (1973). Corner frequencies of $P$ and $S$ waves and models of earthquake sources, Bull. Seism. Soc. Am. 63, 2091-2104.

\author{
SEISMOLOGICAL LABORATORY \\ California Institute of Technology \\ Pasadena, California 91109 \\ Contribution No. 2505
}

Manuscript received June 7, 1974. 